\title{
Robust Registration of Multispectral Satellite Images based on Structural and Geometrical Similarity
}

\author{
Guohua Lv, Qiang Chi, Mohammad Awrangjeb, Jian Li
}

\begin{abstract}
Accurate registration of multispectral satellite images is a challenging task due to the significant and nonlinear radiometric differences between these data. To address this problem, this paper explores the strategy of geometrical similarity between triplets of feature points, and it is combined with the structural similarity between images in a feature-based image registration framework. The underlying principle is that the structural and geometrical similarities generally preserve across the images being registered. In this feature-based image registration framework, a set of control points are firstly detected. Then, the geometric similarity between triplets of control points is defined, followed by a ranking operation of these triplets of control points. The highly-ranked triplets are used to estimate a spatial transformation between images. Finally, initial matches obtained by a benchmark registration technique are refined by the estimated transformation. The experimental results demonstrate the great effectiveness of the proposed technique for registering multispectral satellite images.
\end{abstract}

\section{INTRODUCTION}

$\mathbf{I}$ MAGE registration is a fundamental task in computer vision and image processing applications. It aims to find the correct spatial alignment between images of the same scene that have been acquired in different imaging conditions [1], [17], [18]. The images being registered may be captured at different times, from different viewpoints, by different types of sensors, etc [17], [18].

Image registration methods can be classified into intensitybased [3]-[5] and feature-based [6]-[8], [14], [17] ones. A recent and comprehensive literature review can be found in $\mathrm{Ma}$ et al. [2]. Intensity-based methods compare intensity patterns between images via correlation metrics, while featurebased methods find correspondences between image features such as points, lines and contours. Compared to the featurebased methods, the limitations of the intensity-based methods mainly lie in difficulties in dealing with large content differences and low-overlapping between images, as well as large computational cost caused by the optimization process [18]. In the domain of remote sensing image registration, there

Guohua Lv and Qiang Chi are with the School of Computer Science and Technology, Qilu University of Technology (Shandong Academy of Sciences), Jinan 250353, China (e-mail: guohualv@qlu.edu.cn; 17865151132@163.com).

Mohammad Awrangjeb is with the School of Information and Communication Technology, Griffith University, Nathan, QLD 4111, Australia (e-mail: m.awrangjeb@griffith.edu.au).

Jian $\mathrm{Li}$ is with the School of Cyber Security, Qilu University of Technology (Shandong Academy of Sciences), Jinan 250353, China (Corresponding author, e-mail: scs_lijian@qlu.edu.cn). are some recent developments [6]-[8]. In Chang et al. [6], the most popular local image feature Scale Invariant Feature Transform (SIFT) is improved by computing feature slope and grouping feature points. A pixel-wise representation is used to capture structural and shape properties, and the matching process is speeded up using the three-dimensional Fast Fourier Transform (3DFFT) [7]. In Jiang et al. [8], putative feature correspondences are firstly established by matching local descriptors, followed by removing outliers using geometrical consistency priori together with filtering and denoising theory.

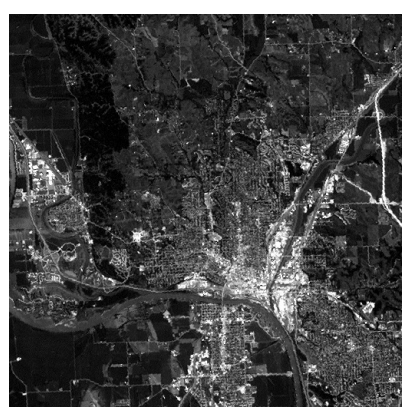

(a)

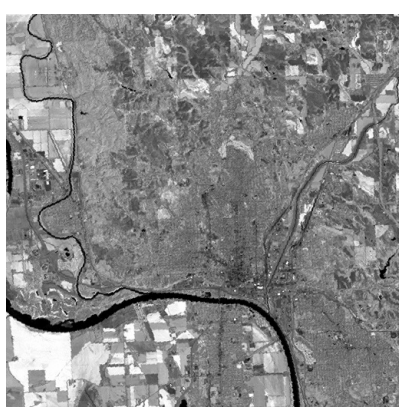

(b)
Fig. 1. A typical pair of multispectral satellite images. The two images shown in (a) and (b) are bands 2 and 4 of Sioux city, Iowa, United States.

This paper focuses on feature-based registration of multispectral satellite images. The central difficulty lies in the significant and nonlinear radiometric (intensity) differences between images [9], [10], [15]-[18]. Fig. 1 shows a typical pair of multispectral satellite images. To achieve robust image registration, the fundamental philosophy is that the structural and geometrical patterns generally preserve across images. Thus, the goal of this paper is to develop an effective registration technique that is robust to significant and nonlinear intensity differences between multispectral satellite images.

Motivated by the fact that phase congruency is invariant to illumination and contract changes, a feature descriptor called Histogram of Oriented Phase Congruency (HOPC) was proposed to address the significant and nonlinear radiometric (intensity) differences in registering multimodal remote sensing images [9]. The limitations of HOPC include the following two aspects. First, the effectiveness of HOPC is likely to be undermined in the cases where similar structures occur within one image. Second, the HOPC descriptors only encode structural properties of images in frequency domain, which does not take into account the image information in 
spatial domain. To address these two issues, we explore the geometrical information between triplets of feature points, which is complementary to the structural information encoded by HOPC, and integrate them into a registration framework. By considering the geometrical information between feature points within one image, the aforementioned first issue can be confidently addressed.

\section{Methodology}

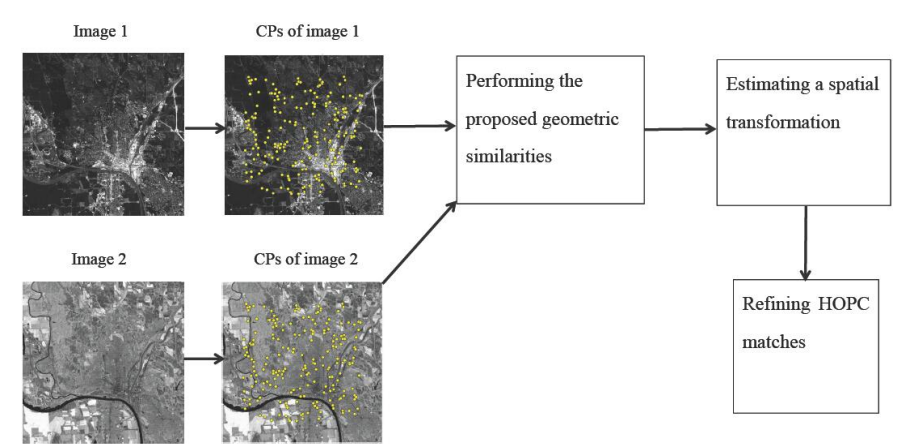

Fig. 2. The framework of the proposed Structural and Geometrical Similarity (SGS). The image pair used for the framework is the same used in Fig. 1.

This section elaborates the proposed technique called Structural and Geometrical Similarity (SGS). An overview of SGS is first given, followed by a few key issues in detail.

\section{A. Overview of $S G S$}

The main steps of the SGS framework illustrated in Fig. 2 are:

1) Given two images to be registered, control points (CPs) are detected by implementing the HOPC algorithm.

2) Based on the detected CPs, the geometrical similarity between triplets of CPs is explored, including the Angle Difference between Triangular Vertices, the Length Ratio of Line Segments, any two Line Segments and their Intersection Angle, and the Orientation of Pairwise Control Points (see Section II-B).

3) Triplets of CPs are ranked and a set of highly-ranked CPs are used to estimate a spatial transformation between the two images (see Section II-C).

4) The estimated transformation is used to evaluate the correctness of the matching results that have been achieved by the HOPC algorithm, thereby generating the final matched CPs (see Section II-D).

\section{B. Proposed Geometrical Similarity}

Let $N$ be the number of pairs of matching CPs between a query image and a target image. Corresponding to these $N$ point pairs, let $P_{q}^{i}$ and $P_{t}^{i}(1 \leq i \leq N)$ denote a pair of CPs in the two images being registered. $P_{q}^{i} \mapsto P_{t}^{i}$ is used to denote a point match from the query image to the target image.

With $N$ point matches, there exist $C_{N}^{3}$ combinations of point triplets in both of query and target images. As $N$ increases, the number of point triplets $C_{N}^{3}$ goes up dramatically. To ensure that the computational cost of the proposed technique

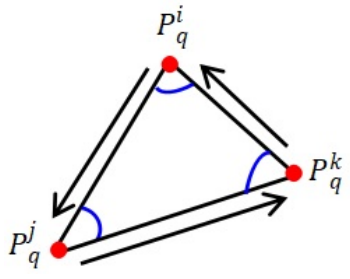

(a)

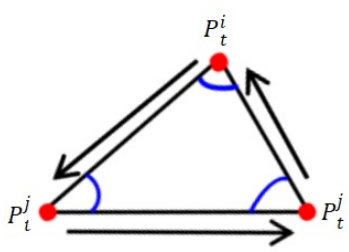

(b)
Fig. 3. Illustrating the proposed geometrical similarity. (a) and (b) are drawn for query image and target image, respectively. A triangular vertex represents a control point. A blue arc between two line segments denotes the angle at each triangle vertex. The orientation of pairwise control points is indicated by the arrow beside the associated line segment.

is acceptable, it is critical to limit the number of point triplets. Fortunately, in order to evenly detect CPs, the block-based Harris operator [13] used in the HOPC algorithm imposes a reasonable restriction on the number of interest points. In order to ensure a low computational cost, the parameters for the block-based Harris operator are finely tuned. Based on the HOPC matching results, three point matches, e.g., the $i^{t h}, j^{t h}$ and $k^{t h}$ matches, lead to a pair of point triplets. For the referencing purpose, this pair of point triplets is denoted as $\left(P_{q}^{i}, P_{q}^{j}, P_{q}^{k}\right) \mapsto\left(P_{t}^{i}, P_{t}^{j}, P_{t}^{k}\right)$ from query image to target image. Fig. 3 illustrates such a pair of point triplets.

With the point triplets generated above, the following four types of geometrical similarity are explored, including the Angle Difference between Triangular Vertices, the Length Ratio of Line Segments, the Line Segments and their Intersection Angle, and the Orientation of Pairwise Control Points. First, the Angle Difference between Triangular Vertices (ADTV) is formulated as

$$
\max _{1 \leq i \leq N}\left\{\left|\theta_{q v}^{i}-\theta_{t v}^{i}\right|,\left|\theta_{q v}^{j}-\theta_{t v}^{j}\right|,\left|\theta_{q v}^{k}-\theta_{t v}^{k}\right|\right\} \leq \varepsilon_{1},
$$

where $\theta_{q v}^{i}, \theta_{q v}^{j}$ and $\theta_{q v}^{k}$ denote the angle at each triangle vertex in the query image, $\theta_{t v}^{i}, \theta_{t v}^{j}$ and $\theta_{t v}^{k}$ stand for the angle at each triangle vertex in the target image, and $\varepsilon_{1}$ is a pre-defined error threshold for the angle difference between corresponding triangular vertices.

Second, the Length Ratio of Line Segments (LRLS) is formulated as

$$
\sigma\left\{\frac{\left|\overrightarrow{P_{q}^{i} P_{q}^{j}}\right|}{\left|\overrightarrow{P_{t}^{i} P_{t}^{j}}\right|}, \frac{\left|\overrightarrow{P_{q}^{i} P_{q}^{k}}\right|}{\left|\overrightarrow{P_{t}^{i} P_{t}^{k}}\right|}, \frac{\left|\overrightarrow{P_{q}^{j} P_{q}^{k}}\right|}{\left|\overrightarrow{P_{t}^{j} P_{t}^{k}}\right|}\right\} \leq \varepsilon_{2},
$$

where $\left|\overrightarrow{P_{q}^{i} P_{q}^{j}}\right|,\left|\overrightarrow{P_{q}^{i} P_{q}^{k}}\right|$ and $\left|\overrightarrow{P_{q}^{j} P_{q}^{k}}\right|$ represent the length of three line segments in the query image, $\left|\overrightarrow{P_{t}^{i} P_{t}^{j}}\right|,\left|\overrightarrow{P_{t}^{i} P_{t}^{k}}\right|$ and $\left|\overrightarrow{P_{t}^{j} P_{t}^{k}}\right|$ denote the length of three line segments in the target image, $\sigma$ is used to compute the standard deviation of the length ratios of corresponding line segments, and $\varepsilon_{2}$ is a predefined error threshold for this standard deviation.

Third, the Line Segments and their Intersection Angle (LSIA) is formulated as

$$
\left\{\begin{array}{l}
\left|\frac{\left|\overrightarrow{P_{q}^{i} P_{q}^{j}}\right|}{\vec{P}}\right| \frac{\left|\overrightarrow{P_{q}^{j} P_{q}^{k}}\right|}{\left|\overrightarrow{P_{t}^{i} P_{t}^{j}}\right|}-1 \mid \leq \varepsilon_{3}, \\
\left|\theta_{q v}^{j}-\theta_{t v}^{j}\right| \leq \varepsilon_{4},
\end{array}\right.
$$


where the first condition means that the length ratio of the corresponding line segments should be close, the second condition says that the angle difference between the corresponding triangular vertices should be reasonably small, both of the two conditions must be simultaneously satisfied, and $\varepsilon_{3}$ and $\varepsilon_{4}$ are pre-defined error thresholds for the two conditions. In Eq. 3, $\overrightarrow{P_{q}^{i} P_{q}^{j}}, \overrightarrow{P_{q}^{j} P_{q}^{k}}$ are selected from the query image, and the corresponding line segments are selected from the target image. In practice, there are two other choices for selecting line segments, as shown in Fig. 3.

Fourth, the Orientation of Pairwise Control Points (OPCP) is formulated as

$$
\sigma\left\{\left|\overrightarrow{P_{q}^{i} P_{q}^{j}}-\overrightarrow{P_{t}^{i} P_{t}^{j}}\right|,\left|\overrightarrow{P_{q}^{j} P_{q}^{k}}-\overrightarrow{P_{t}^{j} P_{t}^{k}}\right|,\left|\overrightarrow{P_{q}^{k} P_{q}^{i}}-\overrightarrow{P_{t}^{k} P_{t}^{i}}\right|\right\} \leq \varepsilon_{5},
$$

where $\overrightarrow{P_{q}^{i} P_{q}^{j}}, \overrightarrow{P_{q}^{j} P_{q}^{k}}$ and $\overrightarrow{P_{q}^{k} P_{q}^{i}}$ represent the orientation of pairwise control points in query image, $\overrightarrow{P_{t}^{i} P_{t}^{j}}, \overrightarrow{P_{t}^{j} P_{t}^{k}}$ and $\overrightarrow{P_{t}^{k} P_{t}^{i}}$ denote the orientation of pairwise control points in target image, $\sigma$ is to compute the standard deviation of orientation differences of corresponding pairwise control points, and $\varepsilon_{5}$ is a pre-defined error threshold for this standard deviation. In Fig. 3, the arrow alongside each line segment indicates the orientation of the associated pairwise control points.

\section{Estimating Spatial Transformation}

The thresholds of Eqs. 1-4, i.e., $\varepsilon_{1}$ to $\varepsilon_{5}$, are used to measure the geometric similarity of triplets of control points. Consistently, the more geometrically similar the compared triplets of CPs are, the smaller such a threshold is. There would be a high probability that those highly-ranked triplets of CPs are exactly corresponding, which perfectly satisfies the conditions expressed in Eqs. 1-4.

Therefore, all compared point triplets are ranked in an ascending order according to their similarity. From the list of ranking, $N_{t}$ pairs of top-ranking point triplets are selected. A top-ranking pair of point triplets $\left(P_{q}^{i}, P_{q}^{j}, P_{q}^{k}\right) \mapsto\left(P_{t}^{i}, P_{t}^{j}, P_{t}^{k}\right)$ means that the associated three point pairs are likely to be correctly matched. These top-ranking point triplets correspond to a set of control points, thereby being used to estimate a spatial transformation between the two images being registered. The Matlab built-in function cp2tform is used to estimate the transformation. It should be noted that projective transformation is used and it is applicable to the satellite images tested in our experiments. Other types of spatial transformation may be adopted.

\section{Evaluating the Correctness of HOPC Matches}

Let $T_{e}$ denote the estimated spatial transformation between the two images, $\left(x_{q}, y_{q}\right) \mapsto\left(x_{t}, y_{t}\right)$ represent a point match from query image to target image that has been decided by the HOPC algorithm. The correctness of $\left(x_{q}, y_{q}\right) \mapsto\left(x_{t}, y_{t}\right)$ can be evaluated by

$$
T_{e}\left[\begin{array}{c}
x_{q} \\
y_{q} \\
1
\end{array}\right]=\left[\begin{array}{c}
\omega x_{t e} \\
\omega y_{t e} \\
\omega
\end{array}\right]
$$

and

$$
\sqrt{\left|x_{t e}-x_{t}\right|^{2}+\left|y_{t e}-y_{t}\right|^{2}} \leq \varepsilon_{p},
$$

where $\left(x_{t e}, y_{t e}\right)$ is the transformed coordinate of $\left(x_{q}, y_{q}\right)$, and $\varepsilon_{p}$ denotes an acceptable pixel error between the transformed coordinate and the groundtruth. A maximum of 4-pixel error for $\varepsilon_{p}$ is considered to be accepted as a true match [19], [20]. If Eq. 6 is satisfied, $\left(x_{q}, y_{q}\right) \mapsto\left(x_{t}, y_{t}\right)$ is decided as a correct match.

\section{Performance Study}

In this section, the proposed SGS will be evaluated against SIFT (Scale Invariant Feature Transform) [11], MOG-ISSIFT (MOG: Magnitudes and Occurrences of Gradient, IS: Improved Symmetric) [17], HD-MOG-IS-SIFT (HD: Higher Discrimination) [17] and HOPC [9].

\section{A. Dataset Description}

The multispectral satellite image dataset used for our experiments is accessible at https://serc.carleton.edu/ eyesinthesky2/week11/get_to_know_multispectral_imaging. html\#11_download_landsat. This dataset includes seven original satellite images, named lowa, louisiana, mono lake, owens valley, salt lake, santa cruz az and vegas, among which five images have seven bands and the other two images have six bands. With various combinations of different bands, 135 satellite image pairs are formed for the purpose of image registration. Fig. 4 shows three sample image pairs that are randomly selected from the dataset.
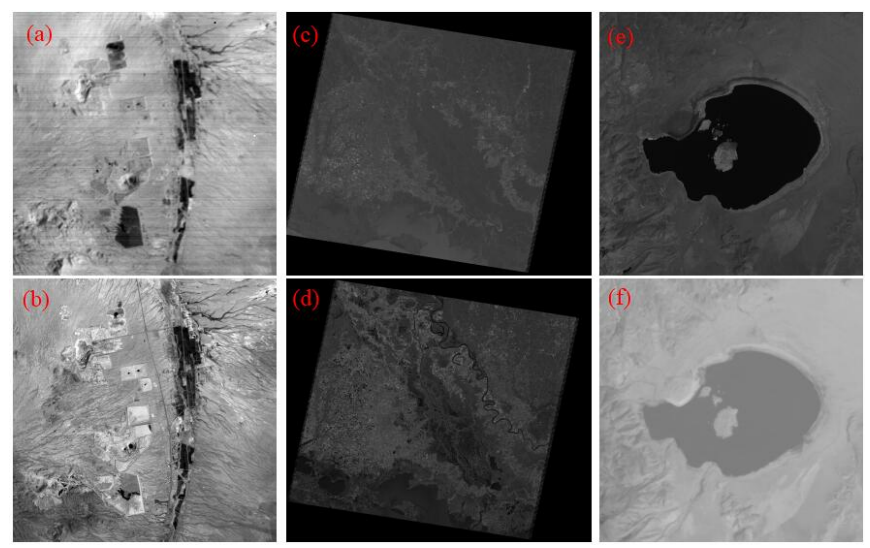

Fig. 4. Sample satellite image pairs.

\section{B. Evaluation Metrics}

The accuracy of an image registration technique depends largely on the matching accuracy. The higher the matching accuracy is, the more accurate the final registration should be [14]-[18]. Hence, the proposed technique is evaluated by

accuracy $=\frac{\text { number of correct matches found }}{\text { number of total matches found }} \times 100 \%$. 
Moreover, recall vs 1-precision [12], [14] is used for performance evaluation. The precision is simply equivalent to the accuracy defined in Eq. 7. The recall is defined as

$$
\text { recall }=\frac{\text { number of correct matches found }}{\text { number of correspondences }} \times 100 \% \text {. }
$$

In addition, the efficiency of the compared techniques will be compared.

\section{Implementation Details}

TABLE I

PARAMETER SETTING FOR SGS

\begin{tabular}{|c|c|c|}
\hline Stage of SGS & Parameters & How Decided \\
\hline Geometrical & $\varepsilon_{1}=5^{\circ}$ & Experimentation \\
Similarity & $\varepsilon_{2}=0.05$ & Experimentation \\
\hline $\begin{array}{c}\text { Geometrical } \\
\text { Similarity }\end{array}$ & $\varepsilon_{3}=0.05 \& \varepsilon_{4}=5^{\circ}$ & Experimentation \\
\hline $\begin{array}{c}\text { Geometrical } \\
\text { Similarity }\end{array}$ & $\varepsilon_{5}=0.05$ & Experimentation \\
\hline $\begin{array}{c}\text { Geometrical } \\
\text { Similarity }\end{array}$ & $N_{t}=3$ & Experimentation \\
\hline $\begin{array}{c}\text { Estimating Spatial } \\
\text { Transformation }\end{array}$ & $\varepsilon_{p}=1.5$ & $\begin{array}{c}\text { Source Code of } \\
\text { [9] }\end{array}$ \\
\hline $\begin{array}{c}\text { Evaluating } \\
\text { Correctness of } \\
\text { HOPC Matches }\end{array}$ & & $\begin{array}{c}\text { Source Code of } \\
\text { [14] }\end{array}$ \\
\hline Recall vs & $T_{l}=0.60 \& T_{u}=0.99$ & Experimentation \\
1-Precision Results & grid_size=[3:1:7] \\
\hline Recall vs 1-Precision & num_points_in_grid=[6:2:10] & \\
\hline
\end{tabular}

Table I lists the parameters which the proposed technique uses at its different stages. Some of these parameter values were decided by experimentation, as indicated in Table I. For the parameters $\varepsilon_{1}$ to $\varepsilon_{5}$, the smaller the value is, the tighter the corresponding condition is. When deciding the parameter $N_{t}$, our consideration is that 3 different pairs of point triplets correspond to at least 5 point pairs that are sufficient for estimating a project transformation. When evaluating the correctness of HOPC matches, the same pixel error is used as Ye et al. [9]. By referring to $\mathrm{Lv}$ et al. [14], the lower and upper limits for the matching threshold are decided so that recall vs 1precision results can be attained for SIFT, MOG-IS-SIFT and HD-MOG-IS-SIFT. The grid_size and num_points_in_grid are tuned from 3 to 7 and from 6 to 10, respectively, so that recall vs 1-precision results can be obtained for HOPC and SGS.

\section{Comparisons in Accuracy and Efficiency}

TABLE II

COMPARISONS IN AVERAGE ACCURACY AND EFFICIENCY OF THE COMPARED TECHNIQUES.

\begin{tabular}{|c|c|c|}
\hline Technique & Average Accuracy & Average Runtime (seconds) \\
\hline SIFT & $74.06 \%$ & 464.80 \\
\hline MOG-IS-SIFT & $84.00 \%$ & 142.35 \\
\hline HD-MOG-IS-SIFT & $87.04 \%$ & 143.33 \\
\hline HOPC & $90.05 \%$ & 43.71 \\
\hline SGS & $98.54 \%$ & 63.48 \\
\hline
\end{tabular}

Table II presents the average accuracy and efficiency achieved by SIFT, MOG-IS-SIFT, HD-MOG-IS-SIFT, HOPC and the proposed SGS. It should be noted that the SGS with the proposed four types of geometrical similarity achieves the same matching accuracy for each image pair. This is because the selected $N_{t}$ pairs of top-ranking point triplets estimate the same spatial transformation, so that the final matched CPs determined by these four types of geometrical similarity are indistinguishable from each other.

By analyzing the results shown in Table II, the following can be concluded.

1) Due to the significant and nonlinear radiometric differences between satellite images, the SIFT is incapable of achieving desirable matching accuracy. This is because SIFT descriptors are relatively sensitive to large content differences between the corresponding parts of images.

2) MOG-IS-SIFT is able to achieve high matching accuracy as compared to SIFT, with an approximate $10 \%$ increase. Based on MOG-IS-SIFT, HD-MOG-IS-SIFT further increases the overall matching accuracy as the HD part plays its role effectively.

3) Overall, HOPC is superior to SIFT, MOG-IS-SIFT and HD-MOG-IS-SIFT, demonstrating that HOPC better deals with the significant and nonlinear radiometric differences between images.

4) The proposed SGS performs best in terms of matching accuracy among the compared techniques, increasing HOPC by $8.49 \%$.

5) When it comes to efficiency, SIFT is the worse efficient in that the largest number of CPs are detected and described among these five compared techniques. The runtime of MOG-IS-SIFT and HD-MOG-IS-SIFT is less than one-third of SIFT's runtime. HD-MOG-IS-SIFT takes slightly more time as compared to MOG-IS-SIFT due to the utilization of the HD part. HOPC is the most efficient, whereas the computational complexity of SGS is nearly 1.5 times that of HOPC. Since the experiments were carried out on Matlab, the efficiency should be significantly improved on some other programming platforms such as $\mathrm{C}$ and/or $\mathrm{C}++$.

Moreover, Fig. 5 compares matching results of HOPC and the proposed SGS. In this example, the proposed SGS achieves a $18.95 \%$ accuracy improvement over HOPC.

\section{E. Comparisons in Recall vs 1-Precision}

The recall vs 1-precision curve is generally plotted for a particular image pair [12], [14] by tuning the matching threshold. Fig. 6 shows the recall vs 1-precision curves for sample image pairs that have been randomly selected from the tested dataset. By analyzing the recall vs 1-precision results, the following conclusions can be drawn.

1) The recall vs 1-precision results achieved by SIFT, MOG-IS-SIFT and HD-MOG-IS-SIFT are almost at the same level.

2) HOPC and SGS outperform SIFT, MOG-IS-SIFT and HD-MOG-IS-SIFT by a large margin. The proposed SGS achieves better recall vs 1-precision as compared to HOPC. 

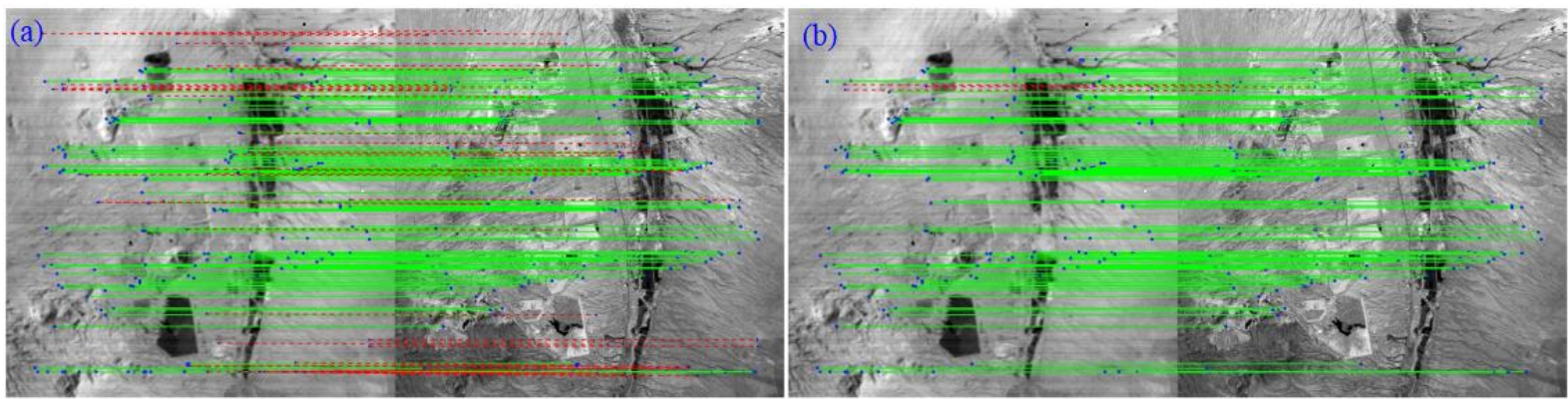

Fig. 5. Comparisons in matching results for a sample image pair. Green (solid) and red (dashed) lines indicate correct and incorrect matches respectively. (a) HOPC: $158 / 200=79.00 \%$. (b) SGS: $143 / 146=97.95 \%$.
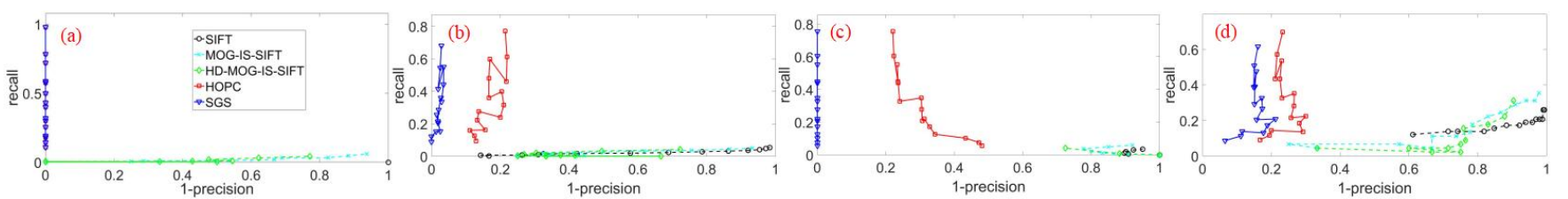

Fig. 6. Recall vs 1-precision curves plotted for sample image pairs. (a): recall vs 1-precision curves for Fig. 1; (b) to (d): recall vs 1-precision curves for Fig. 4(a)-(b), (c)-(d), and (e)-(f), respectively.

\section{CONCLUSION}

This paper has presented a feature-based image registration technique called the SGS by utilizing the structural and geometrical similarity between images. The structural similarity part is based on HOPC descriptors that encode the structural information in the frequency domain, whereas the geometrical similarity part is related to triplets of feature points that belongs to the image information in the spatial domain. The experimental results have demonstrated that the proposed SGS is robust to the significant and nonlinear radiometric differences between multispectral satellite images and achieves state-of-the-art performance in both matching accuracy and recall. In our future work, it is worth integrating more local features in the frequency and spatial domains into the SGS, thereby further enhancing its effectiveness and robustness. Moreover, the SGS will be improved to achieve scale and rotation invariance, and will be extensively evaluated against popular deep learning-based image registration techniques.

\section{REFERENCES}

[1] B. Zitova and J. Flusser, "Image registration methods: A survey," Image Vis. Comput. (IVC), vol. 21, no. 11, pp. 977-1000, Oct. 2003. 1

[2] J. Ma, X. Jiang, A. Fan, J. Jiang J and J. Yan, "Image matching from handcrafted to deep features: A survey," Int. J. Comp. Vis. (IJCV), vol. 129, no. 1, pp. 23-79, Jan. 2021. 1

[3] A. Myronenko and X. Song, "Intensity-based image registration by minimizing residual complexity," IEEE Trans. Med. Imag. (TMI), vol. 29, no. 11, pp. 1882-1891, Nov. 2010. 1

[4] A. Valsecchi, S. Damas, J. Santamaria and L. M. Kacem, "Intensity-based image registration using scatter search," Artif. Intell. Med. (AIM), vol. 60, no. 3, pp. 151-163, Mar. 2014. 1

[5] H. Song and P. Qiu, "Intensity-based 3D local image registration," Pattern Recognit. Lett. (PRL), vol. 94, pp. 15-21, Jul. 2017. 1

[6] H. H. Chang, G. L. Wu and M. H. Chiang, "Remote sensing image registration based on modified SIFT and feature slope grouping," IEEE Geosci. Remote Sens. Lett. (GRSL), vol. 16, no. 9. pp. 1363-1367, Mar. 2019. 1
[7] Y. Ye, L. Bruzzone, J. Shan, F. Bovolo and Q. Zhu, "Fast and robust matching for multimodal remote sensing image registration", IEEE Trans. Geosci. Remote Sens. (TGRS), vol. 57, no. 11, pp. 9059-9070, Jul. 2019. 1

[8] X. Jiang, J. Ma, A. Fan, H. Xu, G. Lin, T. Lu and X. Tian, "Robust Feature Matching for Remote Sensing Image Registration via Linear Adaptive Filtering", IEEE Trans. Geosci. Remote Sens. (TGRS), vol. 59, no. 2, pp. 1577-1591, Feb. 2021. 1

[9] Y. Ye, J. Shan, L. Bruzzone and L. Shen, "Robust registration of multimodal remote sensing images based on structural similarity", IEEE Trans. Geosci. Remote Sens. (TGRS), vol. 55, no. 5, pp. 2941-2958, May 2017. $1,3,4$

[10] J. A. Lee, J. Cheng, et al, "A Low-dimensional Step Pattern Analysis Algorithm with Application to Multimodal Retinal Image Registration," in Proc. CVPR, Jun. 2015, pp. 1046-1053. 1

[11] D. G. Lowe, "Distinctive image features from scale-invariant keypoints," Int. J. Comput. Vis. (IJCV), vol. 60, no. 2, pp. 91-110, Nov. 2004. 3

[12] K. Mikolajczyk and C. Schmid, "A performance evaluation of local descriptors," IEEE Trans. Pattern Anal. Mach. Intell. (TPAMI), vol. 27, no. 10 , pp. $1615-1630$, Oct. 2005. 4

[13] Y. Ye and J. Shan, "A local descriptor based registration method for multispectral remote sensing images with non-linear intensity differences", ISPRS J. Photogramm. Remote Sens. (P\&RS), vol. 90, pp. 83-95, Apr. 2014. 2

[14] G. Lv, S. W. Teng and G. Lu, "Enhancing image registration performance by incorporating distribution and spatial distance of local descriptors," Pattern Recognit. Lett. (PRL), vol. 103, pp. 46-52, Feb. 2018. $1,3,4$

[15] G. Lv, S. W. Teng and G. Lu, "COREG: A corner based registration technique for multimodal images," Multimedia Tools Appl. (MTAP), vol. 77, no. 10, pp. 12607-12634, May 2018. 1, 3

[16] G. Lv, S. W. Teng and G. Lu, "A detector of structural similarity for multi-modal microscopic image registration," Multimedia Tools Appl. (MTAP), vol. 77, no. 6, pp. 7675-7701, Mar. 2018. 1, 3

[17] G. Lv, S. W. Teng and G. Lu, "Enhancing SIFT-based image registration performance by building and selecting highly discriminating descriptors," Pattern Recognit. Lett. (PRL), vol. 84, pp. 156-162, Dec. 2016. 1, 3

[18] G. Lv, "Robust and effective techniques for multi-modal image registration," Ph.D dissertation, Gippsland School Inf. Technol., Monash Univ., Melbourne, VIC, Australia, 2015. 1, 3

[19] M. T. Hossain, G. Lv, S. W. Teng, G. Lu and M. Lackmann, "Improved Symmetric-SIFT for Multi-modal Image Registration," in Proc. DICTA, Dec. 2011, pp. 197-202. 3

[20] G. H. Yang, C. V. Stewart, M. Sofka and C.-L. Tsai, "Registration of challenging image pairs: initialization, estimation, and decision," IEEE Trans. Pattern Anal. Mach. Intell. (TPAMI), vol. 29, no. 11, pp. 19731989, Nov. 2007. 3 